\title{
Hybrid origin of a swordtail species (Teleostei: Xiphophorus clemenciae) driven by sexual selection
}

\author{
AXEL MEYER,*WALTER SALZBURGER* and MANFRED SCHARTL† \\ *Department of Biology, Lehrstuhl für Zoologie und Evolutionsbiologie, University Konstanz, 78457 Konstanz, Germany, †Theodor- \\ Boveri-Institut für Biowissenschaften, Physiologische Chemie I, Biozentrum der Universität Würzburg, 79074 Würzburg, Germany
}

\begin{abstract}
The swordlike exaggerated caudal fin extensions of male swordtails are conspicuous traits that are selected for through female choice. Swords are one of only few examples where the hypothesis of a pre-existing bias is believed to apply for the evolution of a male trait. Previous laboratory experiments demonstrated that females prefer males with longer swords and even females from some swordless species show an affiliation for males of sworded species. Earlier phylogenetic studies based on maternally inherited mitochondrial DNA placed the sworded southern swordtail Xiphophorus clemenciae with swordless platies, contradicting its morphology-based evolutionary affinities. The analyses of new nuclear DNA markers now recover its traditional phylogenetic placement with other southern swordtails, suggesting that this species was formed by an ancient hybridization event. We propose that sexual selection through female choice was the likely process of hybrid speciation, by mating of platy females with males of an ancestral swordtail lineage. In artificial crosses of descendent species from the two potential ancestral lineages of $X$. clemenciae the hybrid and backcross males have swords of intermediate lengths. Additionally, mate choice experiments demonstrate that hybrid females prefer sworded males. These experimental lines of evidence make hybridization through xeno-specific sexual selection by female choice the likely mechanism of speciation.
\end{abstract}

Keywords: hybrid speciation, mate choice experiment, nuclear DNA phylogeny, sexual selection, xeno-specific sexual selection, Xiphophorus

Received 27 July 2005; revision accepted 20 October 2005

\section{Introduction}

The concept of sexual selection as a mechanism to promote the evolution of exaggerated male traits that appear detrimental for survival was first proposed by Darwin (1871). Sexual selection depends on the mating success of certain individuals over others of the same sex. Competition for mates can be direct between members of the same sex, or indirect when mate choice is involved (Panhuis et al. 2001). The prevailing form of the latter type of sexual selection is female choice, which involves the preference of females for certain male traits.

For a long time, the swordlike ventral caudal fin elongation of male swordtails of the genus Xiphophorus has served as an important model for the evolution of elaborate male

Correspondence: Axel Meyer, Fax: +49-7531-883018; E-mail: axel.meyer@uni-konstanz.de ornaments via a female's preference for this trait (Darwin 1871; Ryan \& Wagner 1987; Basolo 1990, 1995). It has even been shown that female platyfish, which belong to the same genus as the swordtails but whose males do not possess swords, prefer conspecific males with artificial swords over normal, swordless ones (Basolo 1990). In addition, Xiphophorus females may also use olfactory cues for intraspecific recognition (McLennan \& Ryan 1999). Species of the swordless sister genus Priapella as well as platyfish of the genus Xiphophorus also are one of only few examples for the pre-existing bias hypothesis (Kaneshiro 1976; Ryan 1998), in that females of both show an apparently ancestral mating preference for males with swords, which probably drove the evolution of this exaggerated male trait (Ryan 1990; Basolo 1995). In the traditional phylogenetic hypothesis of the genus Xiphophorus that was largely based on phenotypic traits (Rosen 1979; Rauchenberger et al. 1990) the swordless platy species were placed basal to the 


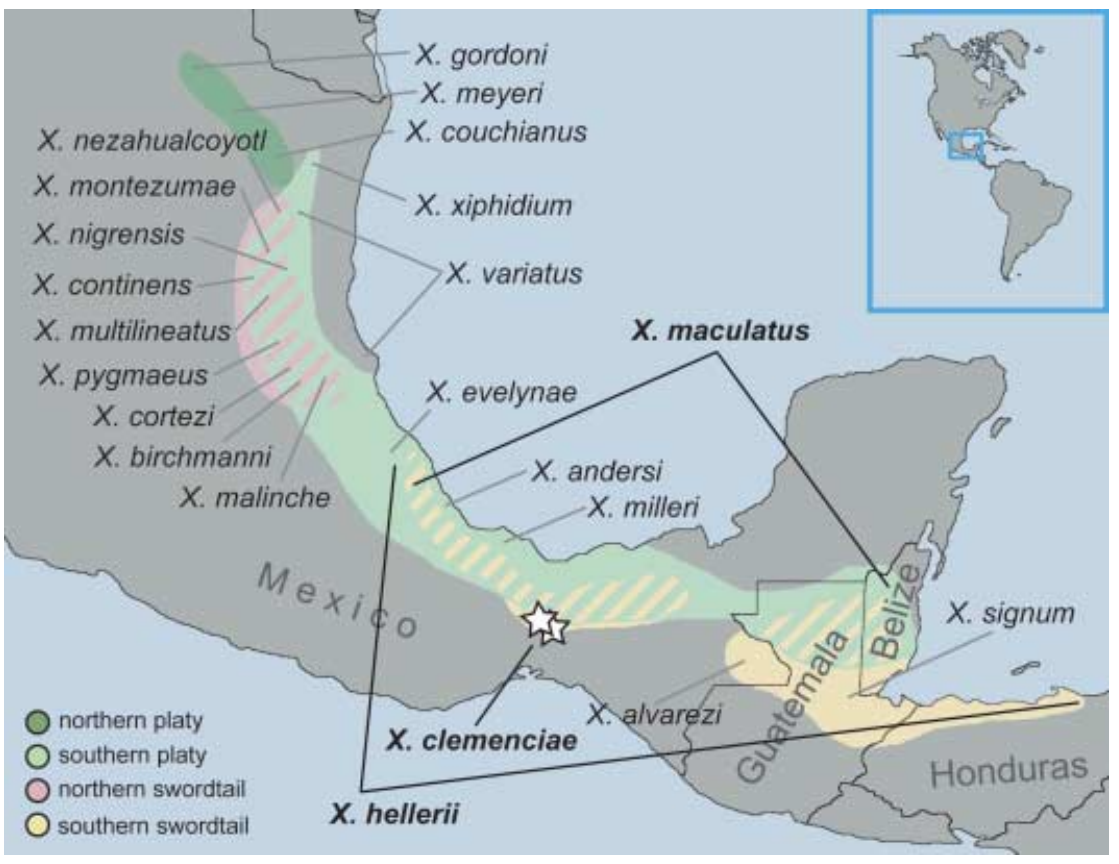

Fig. 1 Map showing the distributions of all 22 Xiphophorus species in Central America. Specimen information and sample localities are contained in the Supplementary material. Species names in bold are those that are most relevant to this study.

sworded swordtail species within this genus. This phylogenetic position of swordless platies would be in support of the hypothesis that a female bias for swords evolved before the male trait itself, possibly also due to 'sensory exploitation' by males of the females' innate sensory bias (Endler \& McLellan 1988; Ryan 1990; Boughman 2002).

With a total of 22 described species, both the northern and southern swordtails and the swordless northern and southern platies comprise the genus Xiphophorus in the species-rich freshwater family Poeciliidae from Central America (Fig. 1). A molecular phylogeny largely based on mitochondrial DNA (mtDNA), which included all described species of the genus (Meyer et al. 1994), disagreed with the traditional phylogeny by placing the northern swordtails as sister group to the southern swordtails plus the platyfish. One notable feature of the mitochondrial phylogeny was the placement of Xiphophorus clemenciae - it had been traditionally assigned to the group of southern swordtails (Rosen 1979; Rauchenberger et al. 1990), but it was, mitochondrially, nested among the southern platyfish (Meyer et al. 1994). One likely explanation for this surprising position of $X$. clemenciae among the southern platies is an ancient hybridization event. However, hardly any nuclear markers, which would allow for further testing of this hypothesis, have been analysed so far.

Here, we report a new phylogeny based on DNA sequences from six nuclear loci with a total length of $3000 \mathrm{bp}$. We also determined additional mtDNA sequences for all species in the genus. While the new mtDNA phylogeny confirms the results of our earlier study (Meyer et al. 1994), X. clemenciae is now, in the nuclear DNA phylogeny, placed among the southern swordtails, which it morpho- logically resembles. This interesting discrepancy in the phylogenetic position of $X$. clemenciae between the mitochondrial and nuclear phylogeny is in agreement with the hypothesis of a hybrid origin of this taxon. To test the hypothesis that the species $X$. clemenciae originated from a hybridization event between swordless female platyfish and sworded male swordtails, we also produced artificial hybrids of two of the potential ancestral lineages of X. clemenciae, namely the southern platyfish Xiphophorus maculatus, and the green swordtail Xiphophorus hellerii. We find that hybrid males and hybrid $\times$ swordtail backcross males have swords of intermediate lengths that resemble those of $X$. clemenciae males. Furthermore, in mate choice experiments, hybrid and backcross females displayed a visual preference for swordtail males, making hybridization through xeno-specific sexual selection - specifically due to a pre-existing (universal) female bias for swords - the likely mechanism for the hybrid origin of the new species.

\section{Materials and methods}

\section{DNA sequence analysis}

Total DNA was extracted from fin clips applying a proteinase $\mathrm{K}$ digestion followed by sodium chloride extraction and ethanol precipitation (Bruford et al. 1998). Polymerase chain reaction (PCR) amplification was performed according to standard methods on an ABI 9700 thermocycler (Applied Biosystems). We used published primers for the amplification of the mitochondrial control region (Meyer et al. 1994; Lee et al. 1995) and for the exon 3 of the nuclear RAG1 gene (Venkatesh et al. 2001). Primers 
Table 1 Sequences of newly designed primers

\begin{tabular}{lll}
\hline DNA locus & Forward primers & Reverse primers \\
\hline D2 & D2-F152: 5'-GATGCAACTTACAGCACTTGA-3' & D2-R488 (see Seckinger et al. 2002) \\
D8 & D8-F91 (see Seckinger et al. 2002) & D8-R500: 5'-GATTTCCACTCAGGACCACTT-3' \\
D29 & D29-F206: 5'-ATTTGTAAAGCCACTGGTTT-3' & D29-R: 5'-TGTATGCTGGTGCAAAGAGCA-3' \\
T36 & T36-F: 5'-TACAGAATGTACAGGAGGCTC-3' & T36-R488 (see Seckinger et al. 2002) \\
\hline
\end{tabular}

for four additional nuclear loci were designed to amplify noncoding flanking regions of the microsatellite loci D2, D8, D29 and T36 (Seckinger et al. 2002) using their original sequences (see Table 1 for primer sequences). One taxon (Xiphophorus maculatus) could not be amplified for D8. Sequences were analysed on an ABI3100 automatic sequencer (Applied Biosystems); sequence data can be obtained from GenBank under the accession numbers listed in Table S1 (Supplementary materials). For each DNA segment, up to eight specimens from two different sampling sites were sequenced for Xiphophorus clemenciae, two to four specimens were analysed for both Xiphophorus hellerii and X. maculatus. For the remaining representatives, one specimen each was used. Two species of the poecilid genus Priapella were included as outgroup (Meyer et al. 1994). Specimen information and sample localities can be obtained from Table S1.

\section{Phylogenetic analyses}

The DNA sequences of the mitochondrial control region (877 bp) were combined with the partial cytochrome $b$ sequences (360 bp) of a previous study (Meyer et al. 1994). DNA sequences of the nuclear genes were combined with previously published X-src sequences (Meyer et al. 1994). The lengths of the nuclear DNA sequences were $394 \mathrm{bp}$ for D2; 515 bp for D8; 381 bp for D29; 394 bp for T36; $1574 \mathrm{bp}$ for RAG1/exon3; and $496 \mathrm{bp}$ for X-src. Single nucleotide polymorphisms at 11 positions in the nuclear DNA segments were coded according to the IUB degenerate base code. The computer program CLUSTAL $w$ (Thompson et al. 1994) was used for sequence alignments, and the alignments were further adjusted by eye. Gaps were encoded as indels.

Prior to phylogenetic analyses we tested the overall phylogenetic signal in each individual nuclear marker and in the combined nuclear data set separately by means of a four-cluster likelihood mapping analysis implemented in PUZZLE 4.0 (Strimmer \& von Haeseler 1997). For the data set combining all nuclear DNA segments, but excluding the outgroup taxa, a percentage fraction of $97.9 \%$ of all possible quartet topologies (7315) were fully resolved, while the percentage fraction of partially resolved and unresolved quartet topologies ranged from $11.4 \%$ (RAG1) to $23.7 \%$ (D29) in the particular nuclear gene segments. This outcome corroborated a strong overall phylogenetic signal in the combined nuclear data set.
For tree reconstruction, we applied maximumparsimony, neighbour-joining, maximum-likelihood and Bayesian inference approaches using the computer programs PAUP ${ }^{*}$ 4.0b10 (Swofford 2003) and MRBAYES 3.0 (http:// morphbank.ebc.uu.se/mrbayes/). We first analysed the individual markers separately, using the general timereversible model of molecular evolution (six classes of substitutions) with a gamma substitution correction and site-specific rates. Due to its greater length compared to the other segments, RAG1 was divided into two parts ranging from alignment positions 1-749 bp and 750-1574 bp, respectively. It turned out that only in the data set consisting of the second part of RAG1 was X. clemenciae resolved within a monophyletic cluster consisting of the representatives of the northern and southern platyfish plus Xiphophorus andersi (trees not shown), which might be the result of a recombination event in that gene. We therefore decided to exclude the first RAG1-segment from the combined analysis of the entire data set.

For maximum-likelihood and Bayesian inference of the combined nuclear (excluding the first 749 bp of RAG1) and mitochondrial data sets, we used the general time-reversible model of molecular evolution (six classes of substitutions) with a gamma substitution correction and site-specific rates, all parameters being estimated from the data sets, as suggested by running the MODELTEST 3.06 routine (Posada \& Crandall 1998). MRBAYES was used to run four Metropoliscoupled Monte-Carlo-Markov-Chains for 10 million generations, starting from a random tree and applying a sample frequency of 10 . The data set was partitioned with respect to the different gene segments. In both data sets, $5 \%$ of trees were excluded as burn-in (based on the observation that $-\ln$ likelihoods stabilized after about $5 \%$ of the sampled trees). For maximum parsimony, an unweighted heuristic search was performed with PAUP* (20 replications). We ran quartet-puzzling analyses (10 000 puzzling steps; same model parameters as in the maximum-likelihood tree search), and maximum-parsimony (1000 replicates) and neighbour-joining (5000 replicates) bootstraps for both the mtDNA and nuclear DNA data sets. We finally performed a Shimodaira-Hasegawa test under a resampling-estimated log-likelihood (RELL) with 1000 bootstrap replicates (as implemented in PAUP $^{*}$ ), in order to test whether the maximum-likelihood topologies based on the nuclear and mtDNA data sets are significantly different. 


\section{Morphology}

The sword index (sword length/standard length) was determined from sexually mature, adult males. For measurements, males were raised individually until all secondary sexual characters were fully developed. X. hellerii and X. clemenciae juvenile males were taken from large population tanks in which laboratory stocks are bred. These stocks are derived from collections made at the Rio Lancetilla, Honduras (X. hellerii), and Rio Grande, Oaxaca, Mexico (X. clemenciae). As developmental endpoint and therefore relevant ontogenetic stage of measurement, the development of the complex bony elements of the gonopodium was determined. Although growth in males ceases with reaching sexual maturation, we waited at least four more weeks before the sword index was determined. Also, after this period no further growth of the sword occurs (Zander 1967).

\section{Mate choice experiments}

To further demonstrate the plausibility of a hybrid origin of $X$. clemenciae due to sexual selection by female preference, we artificially hybridized two extant representatives of the potential ancestral lineages (the swordless platy $X$. maculatus and the southern swordtail species $X$. hellerii). Hybrid and backcross females were also tested for their mate preferences through standard visual information tests (Ryan \& Wagner 1987), by offering these females the choice between a platy and a swordtail male. $\mathrm{F}_{1}$ hybrids were produced in the laboratory between platyfish (X. maculatus strain Jp163A, WLC\# 1352; origin: Rio Jamapa, Veracruz, Mexico) females and swordtail (X. hellerii strain Db-, WLC\# 1337; origin: Rio Lancetilla, Honduras) males by artificial insemination. Backcross hybrids $\left(\mathrm{BC}_{1}\right)$ were produced by crossing $F_{1}$ hybrid females with swordtail males. The $F_{1}$ males originated from four different $X$. maculatus $/ X$. hellerii matings and the $\mathrm{BC}_{1}$ males from five different matings of $F_{1}$ 's with $X$. hellerii. All fish were kept under standard conditions in the aquarium facilities of the Biocenter, University of Würzburg. Females were raised separately from males.

For female choice experiments, a standard visual information test was applied (Ryan 1990). Experiments were conducted in a $43 \times 16 \times 28 \mathrm{~cm}$ aquarium that was divided into three equally sized sections (test aquarium). To each side, a $15 \times 16 \times 28 \mathrm{~cm}$ aquarium was added that held the stimulus males - to ensure that female choice was based on visual cues only. The sections at each side of the test aquarium were the preference zones, while the central section was the neutral zone. The test fish was able to move freely among the three sections. To initiate a trial, a platyfish and a swordtail male were each placed in one of the outer compartments. Then, one female was placed in a plexiglass cylinder into the test aquarium and allowed to acclimatize for $10 \mathrm{~min}$. After this acclimatization period, the cylinder was removed and the female could swim freely into all three sections of the test aquarium. We recorded the time that the female spent in each section adjacent to the males during 5-min trials. The plexiglass cylinder was then returned, the small tanks with the males switched and the observation repeated to rule out side biases of the tested females. All trials in which the female spent more that $80 \%$ of its total time of both tests on one side were excluded as side biased, and all trials where the female spent less that $66 \%$ of its time in one of the preference zones were excluded because of low motivation. Nine platy / swordtail $\mathrm{F}_{1}$ hybrid females, 28 backcross hybrid females from $\mathrm{F}_{1}$ females mated with swordtails, and 28 swordtail females were tested. The $\mathrm{F}_{1}$ females were tested repeatedly with different pairs of males. At least a 3-day pause was implemented between the tests. Due to a short lifespan one female could not be tested in the full number of trials $(n=15)$. Nonparametric statistics were employed to analyse the data (Wilcoxon test). All $P$ values are two-tailed.

\section{Results}

\section{Swordtail phylogeny}

The determination of new complete mitochondrial control region DNA sequences from all described species of the genus Xiphophorus and two outgroup species confirmed the results of an earlier molecular phylogenetic study that was based on cytochrome $b$ and only partial control region sequences (Meyer et al. 1994). By using the maternally inherited mtDNA markers (1237 bp), again the group of northern swordtails was resolved as sister group to the southern swordtails plus the platyfish (Fig. 2a). The monophyly of three different lineages of Xiphophorus, the platies, the northern and southern swordtails, was supported with high to moderate bootstrap and quartetpuzzling values and Bayesian posterior probabilities in all analyses. Within platies, the southern platies are basal and paraphyletic with respect to the northern platies. The phylogenetic position of Xiphophorus andersi could not be resolved conclusively (see also Meyer et al. 1994). This species is typically grouped with the southern platies, but its elongated swordtail-like morphology and its translucent ventral caudal fin extension have made its phylogenetic placement tentative.

Xiphophorus clemenciae - which was traditionally assigned, based on morphological traits, to the southern swordtails (Rosen 1979; Rauchenberger et al. 1990) - was again firmly nested among the southern platyfish (Fig. 2a) as was reported in the initial investigation (Meyer et al. 1994). Uncorrected pairwise distances of the mtDNA sequences determined that Xiphophorus milleri is the closest relative of 

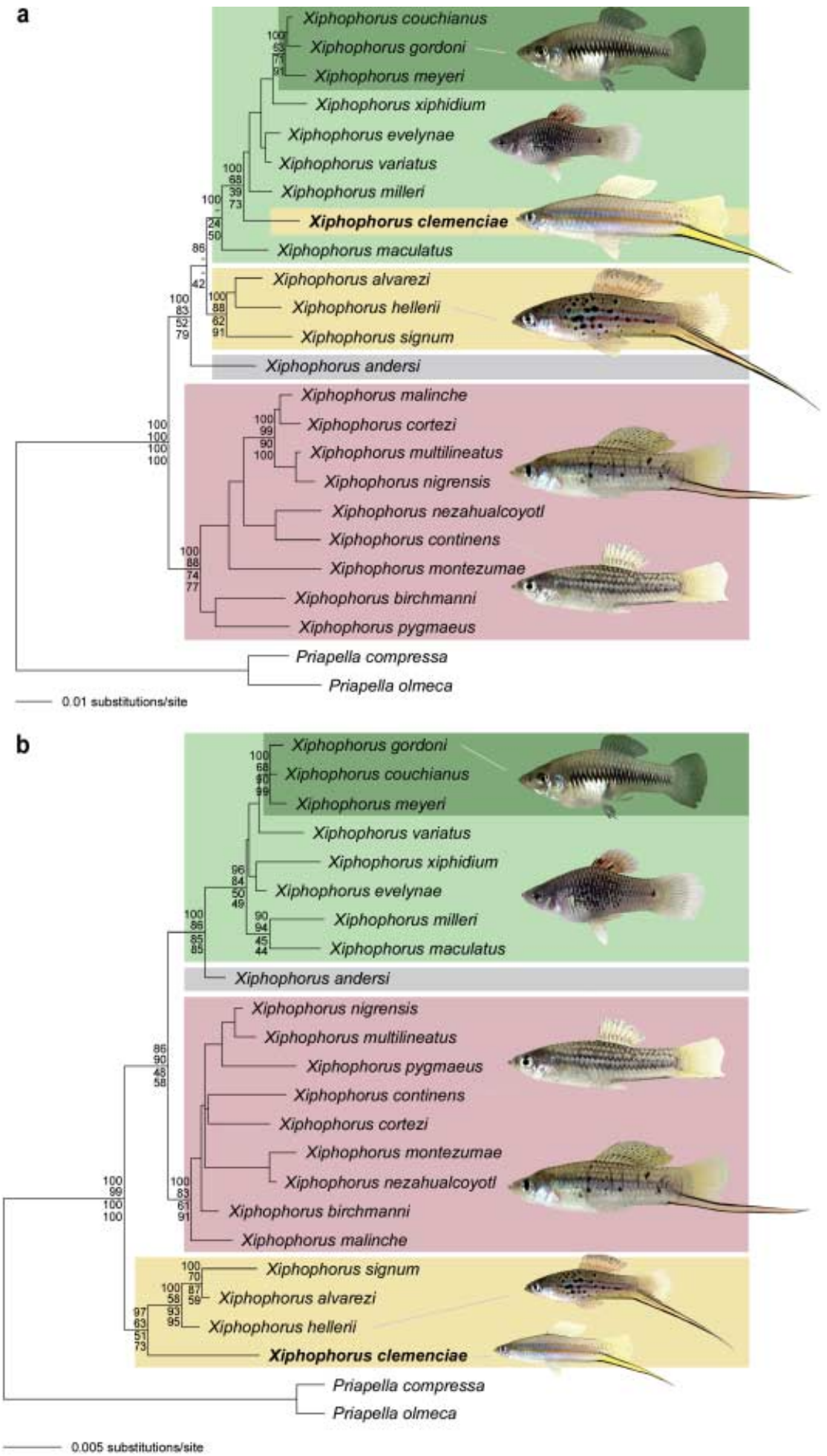

Fig. 2 Phylogeny of all 22 Xiphophorus species using Priapella compressa and Priapella olmeca as outgroup (Meyer et al. 1994). (a) Maximum-likelihood tree based on the complete mitochondrial control region and a segment of the cytochrome $b$ gene (1237 bp). (b) Maximum-likelihood tree based on six nuclear markers (3005 bp). Numbers above the branches are the corresponding Bayesian posterior probabilities and quartet-puzzling values; numbers below the branches represent maximum-parsimony and neighbourjoining bootstrap values. In the mitochondrial phylogeny (a), Xiphophorus clemenciae is nested among the southern platyfish; in the nuclear tree (b) it is grouped together with the southern swordtails instead.
$X$. clemenciae with $2.1 \%$ distance to $X$. clemenciae, compared with $3.1 \%$ to Xiphophorus maculatus, the other southern platy that is genetically close to X. clemenciae. However, $X$. clemenciae differs mitochondrially by at least $3.8 \%$ from other southern swordtail species (Xiphophorus hellerii is its closest relative among the group of southern swordtails in the mtDNA). This amount of DNA sequence divergence from its mitochondrial closest relatives suggests that the origin of $X$. clemenciae is relatively ancient. 

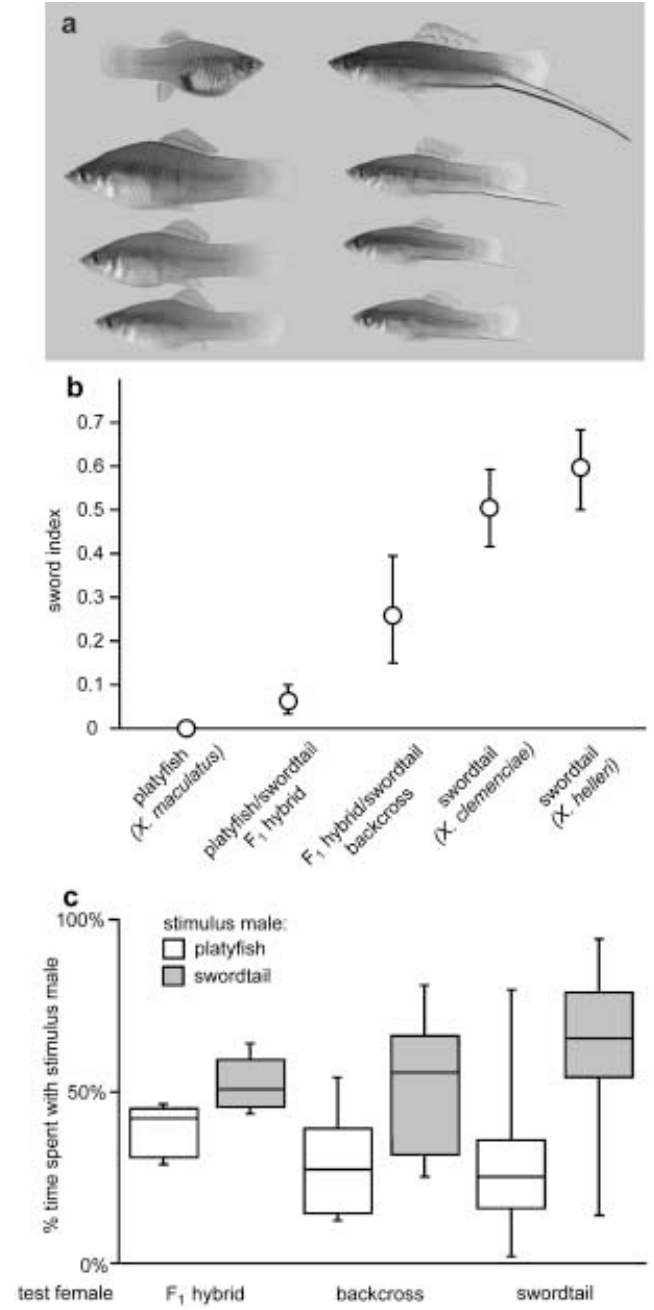

Fig. 3 Laboratory crosses between Xiphophorus maculatus females and males of the swordtail Xiphophorus hellerii. (a) On the left side are females; on the right sides, males. The two fish in the top row are $\mathrm{X}$. maculatus female and a male swordtail $X$. hellerii. Below are $\mathrm{BC}_{1}$ hybrid females and males. (b) Sword index (sword length/ standard length) of male platyfish (X. maculatus), (female) platyfish/ swordtail (X. hellerii) $\mathrm{F}_{1}$ hybrids $(N=14)$, backcross hybrids from $F_{1}$ hybrid females mated with swordtail males (X. hellerii) $(N=108)$, and swordtail (X. hellerii) males $(N=32)$, for comparison the sword index of Xiphophorus clemenciae males $(N=17)$ is shown as well. Bars show standard deviation. (c) To analyse the preference of platyfish/swordtail $F_{1}$ hybrid females, standard visual choice tests were performed. The box plots depict the preferences of platy/swordtail $F_{1}$ hybrid females, backcross hybrid females from $\mathrm{F}_{1}$ females mated with swordtails, and swordtail females. The bar represents the median of the time spent with the stimulus male, the box characterizes the $25 \%$ and $75 \%$ values, respectively. The whiskers describe the $10 \%$ and $90 \%$ values, respectively. There is tendency of $\mathrm{F}_{1}$ hybrid females towards a preference for swordtail males over platy males although not statistically significant $(N=9$, $\mathrm{z}=-1.96, P=0.0499)$. Backcross females from matings of $F_{1}$ hybrid females with swordtail males significantly preferred the swordtail males over platy males $(N=28, z=-2.715, P=0.0066)$. Swordtail females preferred the conspecific males over the males of the platyfish $(N=28, \mathrm{z}=-2.859, P=0.0043)$.
In the phylogeny based on the six nuclear DNA markers (D2, D8, D29, T37, X-src, second part of RAG1), the southern swordtails were consistently resolved as sister group to a clade formed by $X$. andersi plus the platies and the northern swordtails. This branching order was supported by high to moderate bootstrap and quartet-puzzling values and Bayesian posterior probabilities. However, the phylogenetic signal in the nuclear DNA data set was not sufficient to resolve all phylogenetic relationships within the northern and southern platies and the northern swordtails with confidence. The Shimodaira-Hasegawa test confirmed that the nuclear and mitochondrial phylogenies are significantly different $(P<0.05)$. However, it seems that more DNA sequence data are necessary to unambiguously resolve the phylogenetic relationships between the main groups in Xiphophorus.

In contrast to the mtDNA sequences, the majority of the six nuclear markers, when analysed separately, and all combined (but excluding the first section or RAG1) now place X. clemenciae among the southern swordtails (Fig. 2b) as expected based on morphological characters (Rosen 1979; Rauchenberger et al. 1990). X. clemenciae resembles $X$. hellerii, another southern swordtail species, but it is distinguished from the latter species by its shorter sword (Rauchenberger et al. 1990) (see Fig. 3a, b) as well as several other characteristics (e.g. gonopodial structure, colouration of the flanks). In fact, in the nuclear DNA phylogeny, X. clemenciae is most closely related to the southern swordtail $X$. hellerii differing by only $1.0 \%$ sequence divergence in all six nuclear markers combined. Individually, the nuclear markers also support an affiliation of X. clemenciae with the southern swordtails, except for the first 749 bp of RAG1, which place X. clemenciae among the platyfish, most similar to X. milleri and X. maculatus (0.4\% sequence divergence to both). X. clemenciae had identical D2 sequences with X. hellerii and X. alvarezi. It was most similar to X. hellerii based on D29 (0.53\%) and T36 (0.26\%) and to X. alvarezi based on the second part of RAG1 (0.51\%). Based on the X-src marker, $X$. clemenciae was determined to be most similar to the northern swordtail X. birchmanni $(1.01 \%)$ and based on D8 to X. birchmanni and X. malinche (1.23\%). This might be due to the basal position of X. clemenciae among the southern swordtails.

\section{Morphological assessment and mate choice experimensts}

To demonstrate the plausibility of a hybrid origin of $X$. clemenciae due to sexual selection by female preference, we artificially hybridized two extant representatives of the potential ancestral lineages (the swordless platy X. maculatus and the southern swordtail species X. helleri) (Fig. 3a). As has been reported before (Kosswig 1928), the resulting hybrids are fertile, all male hybrids have swords, and the relative length of the sword is shorter in the hybrid 
males compared to the males of one of the parental species (Zander 1975) (see Fig. 3b for measurements of the relative sword length in hybrids and hybrid backcrosses).

Hybrid and backcross females were also tested for their mate preferences through standard visual information tests (Ryan \& Wagner 1987), by offering these females the choice between a platy and a swordtail male. All tests showed, for the first time, that hybrid females of this cross preferred sworded $X$. helleri over nonsworded platy $X$. maculatus males (Fig. 3c). Standard visual choice tests revealed that there is a tendency of $F_{1}$ hybrid females towards a preference for swordtail males over platy males (Fig. 3c). Because of the difficulty in obtaining sufficient numbers of sexually mature $\mathrm{F}_{1}$ females only nine fish could be tested. Therefore, the obtained $P$ values, although significant at the 0.05 level, might need to be interpreted with caution ( $N=9, z=-1.96, P=0.0499)$ (Fig. 3$)$. When these individual females were tested repeatedly, for three of the $\mathrm{F}_{1}$ females the preference for swordtail males was statistically significant $(N=15, \mathrm{z}=-2.229, P=0.0258 ; N=15$, $\mathrm{z}=-2.26, P=0.0238$; and $N=9, \mathrm{z}=-2.1, P=0.0357)$. The remaining six $F_{1}$ females were not tested repeatedly. Backcross females from matings of $F_{1}$ hybrid females with swordtail males significantly preferred the swordtail males over platy males ( 28 females were tested, once each; $\mathrm{Z}=-2.715, P=0.0066)$. Also swordtail females preferred their conspecific males over the males of the platyfish (28 females were tested, once each; $\mathrm{z}=-2.859, P=0.0043$ ).

\section{Discussion}

The discrepancy between the position of Xiphophorus clemenciae in the mitochondrial and nuclear phylogeny (Fig. 2) is best explained as the result of a relatively ancient hybridization event between swordless southern platyfish females with males from a sworded southern swordtail species followed by repeated backcrosses of hybrid females with male swordtails. Under such a scenario, the platyfish species would have contributed the maternally inherited mtDNA while the nuclear background would predominantly be derived from the swordtail species. Hence, the nascent species X. clemenciae was placed among the southern platies in the mitochondrial phylogenies (Fig. 2a) (Meyer et al. 1994) and in the first 749 bp of RAG1, while the remaining nuclear markers support its traditional evolutionary affinities as a member of the southern swordtail assemblage (Fig. 2b). Specifically, we suggest that sexual selection through persistent female choice for swords was the likely mechanisms in this case of hybrid speciation, possibly initiated by the existence of a pre-existing bias for this male trait (Basolo 1990, 1995).

The divergent mtDNA and nuclear DNA phylogenies and data on the phylogeography (Figs 1 and 2) suggest that ancestral lineages of the southern swordtails (such as
Xiphophorus hellerii) and of the southern platies (such as Xiphophorus milleri or Xiphophorus maculatus) were the most likely parental lineages for the hybrid species X. clemenciae. $X$. milleri occurs adjacent to the natural localities of X. clemenciae. X. hellerii and X. maculatus not only show the largest distribution of all species but also widely overlap in their ranges (Fig. 1). In contrast, X. clemenciae is a rare and threatened species whose distribution is the smallest of all southern swordtails, restricted to only a small area in the upper reaches of the Rio Cotzacoalcos, suggesting that the proposed hybridization event was local. Alternatively, the present population of $X$. clemenciae might be the relict of a formerly widespread one.

The feasibility of sexual selection-driven hybridization scenario between a swordtail and a platyfish is supported by two additional sets of experiments. First, in laboratory experiments southern platyfish females were found to show mating preferences for conspecific males with artificial swords (Basolo 1990), and, second, X. maculatus and $X$. hellerii can be crossed in captivity, produce viable hybrids, and backcrosses between $\mathrm{F}_{1}$ hybrids and $X$. hellerii produced sworded males resembling $X$. clemenciae (Kosswig 1928; Walter \& Kazianis 2001). Our own experiments with artificially produced hybrids and hybrid backcrosses between the swordless platy X. maculatus and the southern swordtail species $X$. hellerii (Fig. 3) reveal that male hybrids have swords of intermediate length (Fig. 3a, b) and that female hybrids show a mating preference for sworded males (Fig. 3c). Hence, the ancient female bias for swords might be the behavioural mechanism promoting the hybridization of female platyfish and male swordtails. Hybridization has been considered important in plant evolution (Arnold 1997; Rieseberg 1997), but its importance as a mechanism of speciation remains controversial, particularly in animals where the hybrid origin of new species has only been observed in a few cases (see, e.g. Arnold 1997; Dowling \& Secor 1997; Grant \& Grant 1997a; Turelli et al. 2001). A popular scenario, introgressive hybridization, assumes an initial hybrid population that is interfertile with one or both parental species, which would allow for gene flow from parents to hybrids via backcrossing (Anderson 1949; Arnold 1997). The resulting hybrids then contain a complex mixture of parental genes, whose composition would depend on the actual pathways of introgression (Rieseberg \& Wendel 1993) and range from introgression at a single one locus (e.g. mitochondria) to the reorganization of large portions of the genome (Rieseberg et al. 1995; Dowling \& Secor 1997). In animals, hybridization between closely related species (in geographically defined hybrid zones) has been studied in some detail (see, e.g. Hubbs 1955; Barton \& Hewitt 1989; Scribner 1993; Arnold \& Hodges 1995; Arnold 1997; Grant \& Grant 1997a, b; Good et al. 2000; Epifanio \& Nielsen 2001; Veen et al. 2001; Seehausen 2004), and several cases of a hybrid origin 
of a new animal species have been proposed (see, e.g. Wayne \& Jenks 1991; DeMarais et al. 1992; Dowling \& DeMarais 1993).

Although hybridization in animals is relatively rare, it seems to be somewhat more common in fish (Hubbs 1955; DeMarais et al. 1992; Smith 1992; Dowling \& DeMarais 1993; Epifanio \& Nielsen 2001; Salzburger et al. 2002). However, the (behavioural) mechanism of hybridization is rarely, if ever, known (but see below). This makes the hybrid origin of X. clemenciae particularly interesting, since we offer an explicit mechanism, the xeno-specific preference for swords even among distantly related species within this genus and possibly even beyond (Priapella), the most likely mechanism of hybridization. In the case of $X$. clemenciae, since at least five of the six nuclear loci of the genome of $X$. clemenciae analysed contained only nuclear DNA of the swordtail lineage, backcrosses of the initial hybrid females seem to have occurred predominantly with sexually preferred southern swordtail males. Such a scenario of asymmetric mating is also supported by our mate choice experiments in that we show that both the purebred and the backcross females show significant mating preferences for sworded males.

It has been observed that in nature, hybridization in the genus Xiphophorus is very infrequent (Meyer 1983). The limited distribution of $X$. clemenciae indicates that the hybridization was a rare and locally restricted event that may have only involved a few platy females and, hence, that the platy lineage contributed only few alleles to the novel species. Alternatively, an entire local population of $X$. hellerii might have merged into X. clemenciae, with all other populations of $X$. hellerii being unaffected by the hybridization event. In any case, the nuclear background of southern swordtails would come to dominate over time and account for both, the existence of the long sword and the elongated body form of a typical southern swordtail species, which is already evident in the hybrids (Fig. 3a), and the placement of X. clemenciae among the other species of southern swordtails based on the majority of the nuclear loci as well as morphological traits.

Although other cases of hybrid speciation in fish have been reported (see, e.g. DeMarais et al. 1992; Dowling \& DeMarais 1993; Salzburger et al. 2002), and behavioural mechanisms have been identified that might promote hybridization between two closely related taxa (see, e.g. Brodsky et al. 1988; Ryan \& Rand 1993; McDonald et al. 2001; Rosenfield \& Kodric-Brown 2003; Kodric-Brown \& Rosenfield 2004), this is the first study where sexual selection through female choice is proposed as likely mechanism promoting not only the Fisherian evolution of exaggerated male traits within species, but that this xeno-specific preference was the likely mechanism for the hybrid origin of a new species from more distantly related parental lines. R. A. Fisher stated: 'The grossest blunder in sexual prefer- ence, which we can conceive of an animal making, would be to mate with a species different from its own' (Fisher 1958, p. 144). Apparently, mating preferences may not only prevent hybridization, as is typically thought, but they can also promote the origin of new species (Grant \& Grant 1997b; Rosenfield \& Kodric-Brown 2003). Theory predicts that sexual selection can bring about speciation or maintain mating barriers as well as speed up both the evolution of female choice and male secondary sexual characteristics (Panhuis et al. 2001; Turelli et al. 2001). Hybridization due to more universal sexual preferences might be more prevalent in other groups of animals than previously thought.

If hybrid origins, through sexual selection, of the kind we discovered here, were a more common mechanism for the origin of new species than currently recognized, then the widespread use of maternally inherited mtDNA for phylogenetic studies among closely related species would possibly cause misleading evolutionary inferences.

\section{Acknowledgements}

We are grateful to E. Hespeler for assistance with laboratory work and M. Arnold, T. Dowling, J. Endler, E. Mayr, M. F. Noor, two anonymous reviewers, and Subject Editor M. M. Hansen for comments on the manuscript and valuable discussions. This work was supported by grants of the Deutsche Forschungsgemeinschaft (DFG) (A.M. and M.S.), and a Marie-Curie Fellowship of the EU (W.S.).

\section{Supplementary material}

The supplementary material is available from http://www.blackwellpublishing.com/products/journals/ suppmat/MEC/MEC2810/MEC2810sm.htm

Table S1 Taxonomy information and GenBank accession numbers

\section{References}

Anderson E (1949) Introgressive Hybridization. John Wiley \& Sons, New York.

Arnold ML (1997) Natural Hybridization and Evolution. Oxford University Press, New York.

Arnold ML, Hodges SA (1995) Are natural hybrids fit or unfit relative to their parents? Trends in Ecology EEvolution, 10, 6771.

Barton NH, Hewitt GM (1989) Adaptation, speciation and hybrid zones. Nature, 341, 497-503.

Basolo AL (1990) Female preference predates the evolution of the sword in swordtail fish. Science, 250, 808-810.

Basolo AL (1995) Phylogenetic evidence for the role of a preexisting bias in sexual selection. Proceedings of the Royal Society of London. Series B, Biological Sciences, 259, 307-311.

Boughman JW (2002) How sensory drive can promote speciation. Trends in Ecology \& Evolution, 17, 571-577.

Brodsky LM, Ankney CD, Dennis DG (1988) The influence of male dominance on social interactions in black ducks and mallards. Animal Behaviour, 36, 1371-1378. 
Bruford MW, Hanotte O, Brookfield JFY, Burke T (1998) Multi-locus and single-locus DNA fingerprinting. In: Molecular Analysis of Populations (ed. Hoelzel AR), pp. 283-336. Oxford University Press, New York.

Darwin CR (1871) The Descent of Man, and Selection in Relation to Sex. Murray, London.

DeMarais BD, Dowling TE, Douglas ME, Minckley WL, Marsh PC (1992) Origin of Gila seminuda (Teleostei: Cyprinidae) through introgressive hybridization: implications for evolution and conservation. Proceedings of the National Academy of Sciences, USA, 89, 2747-2751.

Dowling TE, DeMarais BD (1993) Evolutionary significance of introgressive hybridization in cyprinid fishes. Nature, $362,444-$ 446.

Dowling TE, Secor CL (1997) The role of hybridization and introgression in the diversification of animals. Annual Review of Ecology and Systematics, 28, 593-619.

Endler JA, McLellan T (1988) The process of evolution: toward a newer synthesis. Annual Review of Ecology and Systematic, 19, 395-421.

Epifanio J, Nielsen J (2001) The role of hybridization in the distribution, conservation and management of aquatic species. Reviews in Fish Biology and Fisheries, 10, 245-251.

Fisher RA (1958) The Genetic Theory of Natural Selection Dover, New York.

Good TP, Ellis JC, Annett CA, Pierotti R (2000) Bounded hybrid superiority in an avian hybrid zone: effects of mate, diet, and habitat choice. Evolution: International Journal of Organic Evolution, 54, 1774-1783.

Grant PR, Grant BR (1997a) Genetics and the origin of bird species. Proceedings of the National Academy of Sciences, USA, 94, 7768-7775.

Grant PR, Grant BR (1997b) Hybridization, sexual imprinting, and mate choice. American Naturalist, 149, 1-28.

Hubbs CL (1955) Hybridization among fishes in nature. Systematic Zoology, 4, 1-20.

Kaneshiro KY (1976) Ethological isolation and phylogeny in the Planitibia subgroup of Hawaiian Drosophila. Evolution, 30, 740-745.

Kodric-Brown A, Rosenfield JA (2004) Populations of Pecos pupfish (Cyprinodon pecosensis) differ in their susceptibility to hybridize with sheepshead minnow (C. variegatus). Behavioral Ecology and Sociobiology, 56, 116-123.

Kosswig C (1928) Über Kreuzungen zwischen den Teleostiern Xiphophorus helleri und Platypoecilius maculatus. Zeitschrift für ind Abstract - u. Vererbosl., 47, 150-158.

Lee WJ, Conroy J, Howell WH, Kocher TD (1995) Structure and evolution of teleost mitochondrial control regions. Journal of Molecular Evolution, 41, 54-66.

McDonald DB, Clay RP, Brumfield RT, Braun MJ (2001) Sexual selection on plumage and behavior in an avian hybrid zone: experimental tests of male-male interactions. Evolution: International Journal of Organic Evolution, 55, 1443-1451.

McLennan DA, Ryan MJ (1999) Interspecific recognition and discrimination based upon olfactory cues in northern swordtails. Evolution, 53, 880-888.

Meyer M (1983) Xiphophorus-Hybriden aus Nord-Mexiko, mit einer Revision der Taxa X. kosszanderi und IIIX. roseni. Zoologische Abhendlungen Steatliche Naturhistorische Sommlungen Dresden, $38,258-291$

Meyer A, Morrissey JM, Schartl M (1994) Recurrent origin of a sexually selected trait in Xiphophorus fishes inferred from a molecular phylogeny. Nature, 368, 539-542.
Panhuis TM, Butlin R, Zuk M, Tregenza T (2001) Sexual selection and speciation. Trends in Ecology \& Evolution, 16, 364-371.

Posada D, Crandall KA (1998) MODELTEST: testing the model of DNA substitution. Bioinformatics, 14, 817-818.

Rauchenberger M, Kallman KD, Morizot DC (1990) Monophyly and geography of the Rio Panuco Basin swordtails (genus Xiphophorus) with description of four new species. American Museum Novitates, No. 2975, American Museum of Natural History, New York.

Rieseberg LH (1997) Hybrid origins of plant species. Annual Review of Ecologicy and Systematics, 25, 359-619.

Rieseberg LH, Van Fossen C, Desrochers AM (1995) Hybrid speciation accompanied by genomic reorganization in wild sunflowers. Nature, 375, 313-316.

Rieseberg LH, Wendel JF (1993) Introgression and its consequences in plants. In: Hybrid Zones and the Evolutionary Process (ed. Harrison R), pp. 70-109. Oxford University Press, New York.

Rosen DE (1979) Fishes from the uplands and intermontane basins of Guatemala: revisionary studies and comparative geography. Bulletin of the American Museum of Natural History, 162, 268-375.

Rosenfield JA, Kodric-Brown A (2003) Sexual selection promotes hybridization between Pecos pupfish, Cyprinodon pecosensis and sheepshead minnow, C. variegatus. Journal of Evolutionary Biology, 16, 595-606.

Ryan MJ (1990) Sexual selection, sensory systems, and sensory exploitation. Oxford Surveys in Evolutionary Biology, 7, 157-195.

Ryan MJ (1998) Sexual selection, receiver biases, and the evolution of sex differences. Science, 281, 1999-2003.

Ryan MJ, Rand AS (1993) Species recognition and sexual selection as a unitary problem in animal communication. Evolution, 47, 647-657.

Ryan MJ, Wagner WE (1987) Asymmetries in mating preferences between species: female swordtails prefer heterospecific males. Science, 236, 595-597.

Salzburger W, Baric S, Sturmbauer C (2002) Speciation via introgressive hybridization in East African cichlids? Molecular Ecology, 11, 619-625.

Scribner KT (1993) Hybrid zone dynamics are influenced by genotype-specific variation in life-history traits: experimental evidence from hybridizing Gambusia species. Evolution, 47, 632-646.

Seckinger J, Brinkmann H, Meyer A (2002) Microsatellites in the genus Xiphophorus, developed in Xiphophorus montezumae. Molecular Ecological Notes, 2, 4-6.

Seehausen O (2004) Hybridization and adaptive radiation. Trends in Ecology \& Evolution, 19, 198-207.

Smith GR (1992) Introgression in fishes: significance for paleontology, cladistics, and evolutionary rates. Systematic Biology, 41, 41-57.

Strimmer K, von Haeseler A (1997) Likelihood-mapping: a simple method to visualize phylogenetic content of a sequence alignment. Proceedings of the National Academy of Sciences, USA, 94, $6815-6819$.

Swofford DL (2003) PAUP*: Phylogenetic Analyses Using Parsimony (*and Other Methods) Version 4.0. Sinauer Associates, Sunderland, Massachusetts.

Thompson JD, Higgins DG, Gibson TJ (1994) CLUSTAL w: improving the sensitivity of progressive multiple sequence alignment through sequence weighting, position-specific gap penalties and weight matrix choice. Nucleic Acids Research, 22, 4673-4680.

Turelli M, Barton NH, Coyne JA (2001) Theory and speciation. Trends in Ecology \& Evolution, 16, 330-342. 
Veen T, Borge T, Griffith SC et al. (2001) Hybridization and adaptive mate choice in flycatchers. Nature, 411, 45-50.

Venkatesh B, Erdmann MV, Brenner S (2001) Molecular synapomorphies resolve evolutionary relationships of extant jawed vertebrates. Proceedings of the National Academy of Sciences, USA, 98, 11382-11387.

Walter RB, Kazianis S (2001) Xiphophorus interspecies hybrids as genetic models of induced neoplasia. ILAR Journal, 42, 299321.

Wayne RK, Jenks SM (1991) Mitochondrial DNA analysis implying extensive hybridization of the endangered red wolf Canis rufus. Nature, 351, 565-568.

Zander CD (1967) Ökologische und morphologische Beiträge zur Systematik und geographischen Verbreitung der Gattung Xiphohphours (Pisces). Mitteilungen aus dem Hamburgischen Zoologischen Museum und Institut, 64, 57-125.

Zander CD (1975) Genetische Merkmalsanalyse als Hilfsmittel bei der Taxonomie der Zahnkarpfen-Gattung Xiphophorus. Zeitschrift für Zoologische Systematik und Evolutionsforschung, 13, 63-75.

Axel Meyer is head of the Lehrstuhl für Zoologie und Evolutionsbiologie at the University of Konstanz. He is an evolutionary geneticist interested in ecological, developmental and genomic aspects of speciation. The research of Walter Salzburger, a postdoctoral fellow in the lab of Axel Meyer, focuses on the understanding of speciation and adaptive radiation (particularly in East African cichlid fishes). Manfred Schartl is head of the Department of Physiological Chemistry I at the Biocenter of the University of Wuerzburg. His studies focus on melanoma research using the Xiphophorus model, evolution of sex determination and molecular evolution. 